\title{
MicroRNA-4284 inhibits colon cancer epithelial- mesenchymal transition by down-regulating Perilipin 5
}

\author{
Xiaofei MIAO, Zengyao LI, Ye ZHANG*, Tong WANG* \\ Department of General Surgery, Nanjing Medical University Affiliated Wuxi people's Hospital, Wuxi, Jiangsu Province, China \\ *Correspondence: aanti@163.com; zhangye2002520@aliyun.com \\ https://doi.org/10.37175/stemedicine.v2i6.85
}

\begin{abstract}
Background: MicroRNA (miR) has been suggested in the development of several types of cancer; yet, the exact function of miR-4284 in colon cancer remains elusive.
\end{abstract}

Methods: MiR-4284 expression was assessed in normal colon cell line CCD-18Co, and HT-29 and SW480 cell lines representing human colon cancer. Potential target gene of miR-4284 was predicted using TargetScanHuman, and experimentally verified using luciferase report assay. Wound-healing, cell invasion and attachment were evaluated to determine the effect of miR-4284 on the migration, invasion, and metastatic properties of colon cancer cell lines. Expression of epithelial-mesenchymal transition (EMT) phenotypic protein hallmarks, including $\mathrm{N}$-cadherin, E-cadherin, as well as Vimentin, was also evaluated.

Results: MiR-4284 was significantly decreased in colon cancer cell lines, and Perilipin 5 (PLIN5) was found to be directly targeted by miR-4284. Ectopic expression of miR-4284 significantly reduced endogenous expression level of PLIN5 in colon cancer cell lines, suppressing migration, invasion, and metastatic phenotypes. In addition, re-introducing miR-4284 reversed the expression profile of EMT markers.

Conclusion: Our findings for the first time identify miR-4284 as an anti-tumor miRNA in colon cancer, which acts to reduce PLIN5 and inhibit EMT, leading to inhibited colon cancer tumorigenesis. These results highlight the potential of miR-4284 as a therapeutic target in metastatic colon cancer.

Keywords: MicroRNA-4284 - Colon cancer - Perilipin $5 \cdot$ Tumorigenesis · Epithelial-mesenchymal transition

\section{Introduction}

Owing to its lethality, colon cancer causes a significant number of human deaths across the globe. In 2017, approximately 0.1 million new colon cancer cases were reported in US alone (1). Generally, colon cancer treatment includes surgery, chemotherapy and/or radiotherapy (2). However, due to severe side effects of colon cancer chemotherapy, the life quality of the patients is badly impaired (3). Hence, identification of safer drugs and potent therapeutic targets may help to resolve the

Received: Mar 2, 2021; Accepted: Mar 16, 2021.

(C) The Author(s). 2020 This is an Open Access article distributed under the terms of the Creative Commons License (http://creativecommons.org/licenses/by/4.0/) which permits unrestricted use, distribution, and reproduction in any medium or format, provided the original work is properly cited. problem and enable the efficient management of colon cancer.

MicroRNAs (miRNAs, miRs), a subset of non-coding RNAs that can target mRNAs with complementary sequences and result in translational degradation and/ or inhibition (4), are vital players in the proliferation, differentiation, and survival of cells. In 1993, a small RNA was discovered as the first miRNA, transcribed from the lin-4 locus of Caenorhabditis elegans (5). Seven years later, let-7, the first mammalian miRNA, was identified (6). Those two key findings sparked a number of genomic studies, which demonstrated extensive transcription of non-coding RNAs, including miRNAs $(7,8)$.

The capacity of certain miRNAs to target several mRNAs critically modulated in disease states inspires promising therapeutic candidates (miRNA mimics) or 
targets (anti-miRs) (1, 9-11). In the context of colon cancer, one novel mature microRNA, miR-4284, has been seldom studied. While in other types of human cancers, miR-4284 is frequently involved. For instance, in gastric cancer, miR-4284 was found to promote tumorigenicity by targeting ten-eleven translocation 1 (12). Up-regulating miR-4284 in human glioblastoma could inhibit the viability and induce apoptosis of cancer stem-like cells (13). In clear cell papillary renal cell carcinoma, miR-4284 was shown to be one of the down-regulated miRNAs in a miRNA profiling study (14). Of interest to our study, recently in a similar miRNA profiling investigation among a panel of radioresistant prostate cancer cells, miR-4284 was among the most significantly dysregulated miRNAs (15).

In the present study, we sought to uncover the function of miR-4284 in human colon cancer and elucidate its potential molecular target, as well as the signaling pathway(s) involved in its action.

\section{Materials and Methods Cell lines}

HT-29 and SW480 cells (both human colon cancer cell lines), and CCD-18Co cells (a human normal colon cell line) were acquired from the American Type Culture Collection (ATCC). Cells were maintained in complete RPMI-1640 medium (Hyclone; GE Healthcare) with 10\% fetal bovine serum (FBS; Gibco), $100 \mathrm{U} / \mathrm{ml}$ penicillin and $100 \mu \mathrm{g} / \mathrm{ml}$ streptomycin, at $37{ }^{\circ} \mathrm{C}$ in a humidified atmosphere containing 5\% CO2.

\section{MicroRNA assays}

mirVana miRNA negative control (4464061) and hsamiR-4284 mirVana miRNA mimic (MC17194) were both purchased from Thermo Fisher Scientific (Waltham, MA, USA). Transfection of cells was conducted according to the provided protocols. Following transfection, cultures were kept for another $48 \mathrm{~h}$ before any further experiment.

\section{Luciferase reporter assay}

The potential miR-4284 binding site at the 3'-untranslated region (3'-UTR) of Perilipin 5 (PLIN5) was inserted at the downstream of the firefly luciferase open reading frame (ORF) driven by a CMV promoter in a pCMV-Firefly Luc vector (16156; Thermo Fisher Scientific). Luciferase construct containing mutated 3'-UTR was prepared with a standard overlap-extension protocol. To determine the luciferase activity, HT-29 or SW480 cells in a 24-well plate $\left(5 \times 10^{4}\right.$ per well $)$ were co-transfected with $200 \mathrm{ng}$ of wild-type or mutant luciferase constructs, and $400 \mathrm{ng}$ of hsa-miR-4284 or negative control, using Lipofectamine 2000 (Invitrogen). After 48 h, cells were harvested and assessed using the Luc-Screen Extended-Glow Luciferase Reporter Gene Assay System (Thermo Fisher Scientific), following provided protocols.

\section{RNA extraction and quantitative real time polymerase chain reaction (qRT-PCR)}

Extraction of total mRNA was performed with mirVana miRNA Isolation Kit (Thermo Fisher Scientific). The miR4284 expression level was assessed by miRNA-specific TaqMan Pri-miRNA Assay (Hs04227316_pri; Thermo Fisher Scientific) and normalized by RNU48 Control miRNA Assay (001006; Thermo Fisher Scientific). $1 \mu \mathrm{g}$ of total RNA was prepared for reverse-transcription using Superscript II First-Strand Synthesis kit (Thermo Fisher Scientific) at the manufacturer's recommendation. Levels of GAPDH mRNA were used for normalization. Primers used in the current study are presented in Table $\mathbf{1 .}$

\section{Western blot}

Cells were plated in 6 -well plates at $1 \times 10^{6}$ cells/well density with $2 \mathrm{ml}$ completed RPMI-1640 medium. $24 \mathrm{~h}$ following transfection with hsa-miR-4284 or negative control, total protein was prepared with RIPA buffer (150 $\mathrm{mM} \mathrm{NaCl}, 50 \mathrm{mM}$ Tris-HCl pH 7.4, 1 mM EDTA, 1\% sodium deoxycholate, $1 \%$ Triton $\mathrm{X}-100,0.1 \%$ sodium dodecyl sulfate (SDS), and protease inhibitors). Protein quantity was assessed by the BCA assay. Proteins of equal amounts were resolved using SDS-PAGE and then transferred onto a nitrocellulose membrane with the semi-dry transfer unit (Bio-Rad Laboratories). Next, the membrane was incubated in blocking buffer (phosphate buffer saline (PBS) plus $0.1 \%$ Tween-20) with 5\% nonfat milk for $20 \mathrm{~min}$, followed by hybridization with primary antibodies at $4{ }^{\circ} \mathrm{C}$ overnight. Primary antibody against PLIN5 was purchased from Proteintech (\#26951-1-AP; Wuhan, China), antibodies against N-cadherin (ab18203), E-cadherin (ab76055), Vimentin (ab92547), and GAPDH (ab181602) were all commercially available from Abcam (Cambridge, MA, USA). After washes in blocking buffer, membranes were hybridized with HRP-conjugated secondary antibody (Thermo Fisher Scientific) for $1 \mathrm{~h}$ at ambient temperature before visualization with Luminata Forte Western HRP Substrate (EMD Millipore).

\section{Wound-healing assay}

Cells $\left(1 \times 10^{5}\right)$ transfected with hsa-miR-4284 or miRNA

Table 1. Primers used for RT-PCR.

\begin{tabular}{ccc}
\hline Gene & Sense (5' to 3') & Antisense (5' to 3') \\
\hline PLIN5 & AAGGCCCTGAAGTGGGTTC & GCATGTGGTCTATCAGCTCCA \\
TGF- $\beta$ & GGCGATACCTCAGCAACCG & CTAAGGCGAAAGCCCTCAAT \\
GAPDH & CAGCCTCAAGATCATCAGCA & TGTGGTCATGAGTCCTTCCA \\
\hline
\end{tabular}


A

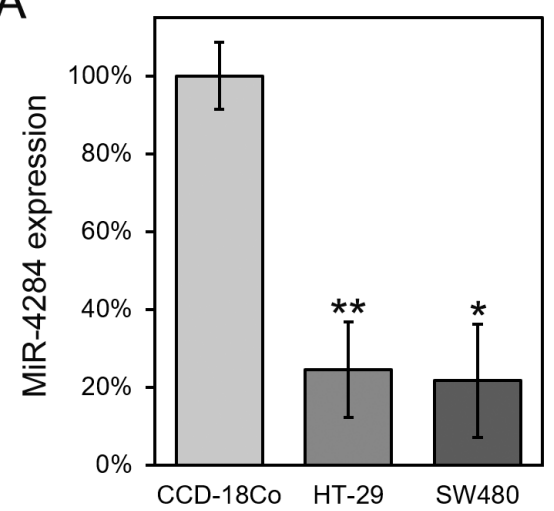

B

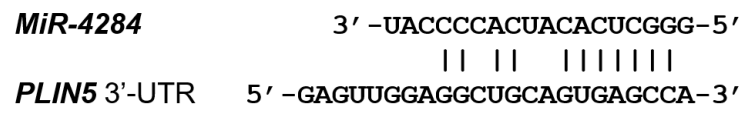

C

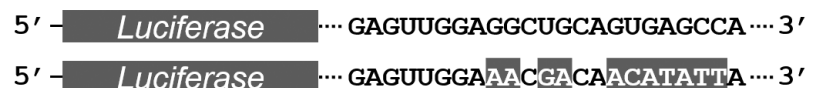

$\mathrm{D}$

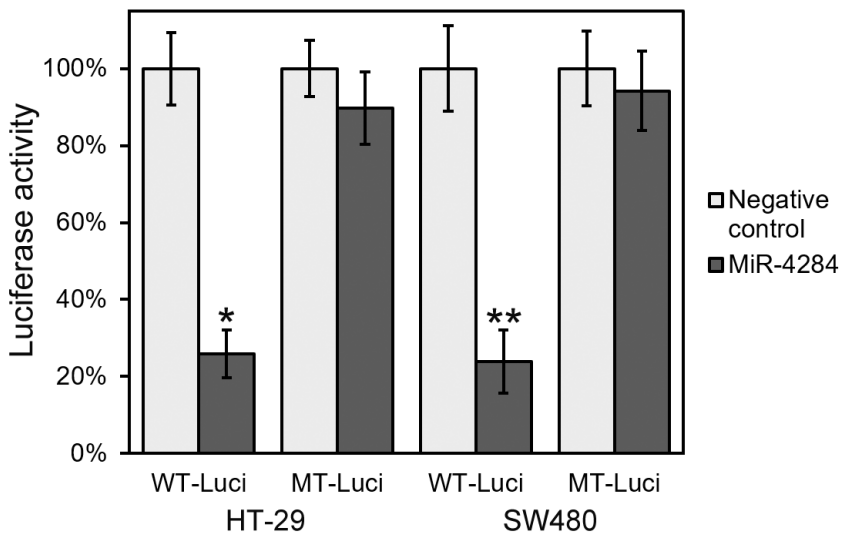

Figure 1. MiR-4284 expression is reduced in colon cancer cells, and directly targets 3'-UTR of PLIN5. (A) MiR-4284 levels in normal human colon cell line CCD-18Co compared with HT-29 and SW480 cells. (B) Sequences of putative miR-4284 binding sites on the 3'-UTR of PLIN5. (C) Schematics of luciferase reporter constructs: top, wild type (WT) luciferase construct (WT-Luci) contains original putative binding site from PLIN5 3'-UTR; bottom, mutated version (MT-Luci). (D) WT and mutated luciferase constructs along with either miR-4284 or miRNA negative control were transfected into HT-29 and SW480 cells. Luciferase activity was presented as relative to respective negative control. Values were mean $\pm S D$ of 3 independent biological replicates; ${ }^{* *} P<0.01,{ }^{*} P<0.05$, compared to respective control.

negative control were seeded into 6-well plate post transfection. A linear cut was generated cautiously across the confluent monolayer cells with the tip of a $100 \mu \mathrm{l}$ sterile pipette, after which gentle washes of PBS were performed to remove the debris. Migration was quantitated as the distance the growing edge moved on the wounded monolayer after $36 \mathrm{~h}$, and migration rates were calculated as the percentages relative to the appropriate control.

\section{Cell invasion assay}

Cell invasion assay was conducted using a 24-well plate with chamber inserts (pore size: $8 \mu \mathrm{m}$, BD Biosciences). The hsa-miR-4284- or miRNA negative controltransfected cells $\left(1 \times 10^{5}\right.$ per well, diluted with serumfree culture medium) were fed into the upper chambers with membranes coated with Matrigel. The lower compartments received $500 \mu$ complete medium per well to attract cells. The cultures were maintained in the incubator at $37^{\circ} \mathrm{C}$ for $24 \mathrm{~h}$, and afterwards treated with 20 $\mu \mathrm{M}$ 5-ethynyl-2'-deoxyuridine (EdU) at $37{ }^{\circ} \mathrm{C}$ for another $4 \mathrm{~h}$. Membrane inserts were then detached for staining with the EdU kit (Invitrogen). For each well, six random microscopic fields were counted, and the percentage of cell numbers relative to the appropriate control was calculated as the invasion rate.

\section{Cell attachment and detachment assay}

The cells $\left(1 \times 10^{5}\right.$ cells per well $)$ were plated in a 24 -well plate. In brief, to assess cell attachment, non-attached cells were removed by two washes with PBS after incubation for $1 \mathrm{~h}$, and the cells that remained attached were trypsinized for counting. The attachment rate was calculated as the percentage of attached cells in total cells and normalized to the appropriate control. To assess detachment, cells were cultured for $24 \mathrm{~h}$ and then treated with $0.05 \%$ trypsin for 3 min to be detached and counted. The remaining cells still attached were further trypsinized with $0.25 \%$ trypsin for counting. The percentage of detached cells in total cells was defined as the detachment rate, normalized to the appropriate control.

\section{Statistical analysis}

All values were expressed as means \pm standard deviations (SDs). Two-tailed student's t-test was employed to examine statistical differences between treatment groups. Differences were regarded as statistically significant with $p$ less than 0.05 .

\section{Results}

\section{MiR-4284 is down-regulated in colon cancer cell lines}

First, with qRT-PCR evaluation of miR-4284 expression in human colon cancer cell lines HT-29 and SW480, we discovered that miR-4284 is markedly lower compared to its levels in the normal colon cell line CCD-18Co (Figure 1A). Next, using the TargenScanHuman webtool (16), we identified that the 3'-UTR of PLIN5 mRNA harbored a putative binding site complementary to miR-4284 (Figure 1B). These findings indicated that miR-4284, which may target PLIN5, was substantially suppressed in colon cancer cells in comparison with normal human colon cells. 
A

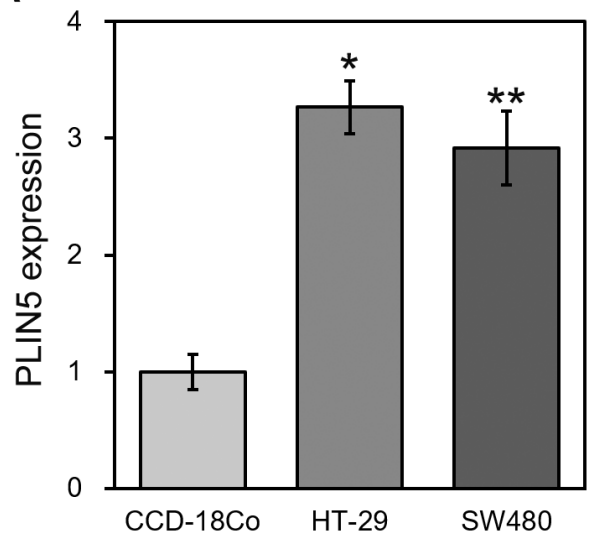

B

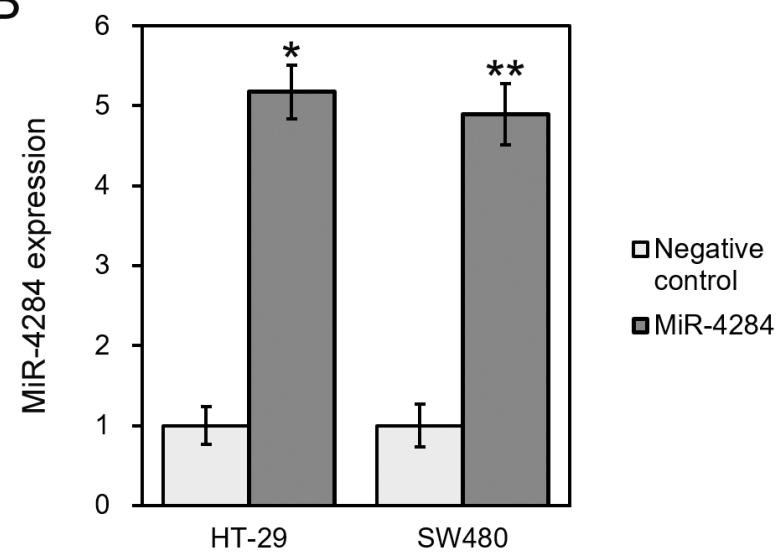

Figure 2. MiR-4284 mimic elevates miR-4284 expression in colon cancer cells. (A) PLIN5 mRNA levels in normal human colon cell line CCD-18Co compared with HT-29 and SW480 cells. (B) Relative miR-4284 levels in HT-29 and SW480 cells after transfection with either miR-4284 mimic or miRNA negative control. Values were mean \pm SD of 3 independent biological replicates; ${ }^{* *} \mathrm{P}<0.01$, * $\mathrm{P}<0.05$, compared to negative control.

\section{MiR-4284 directly binds to the 3'-UTR of PLIN5}

Next, we carried out validation of the putative miR4284binding motif on the 3'-UTR of PLIN5, employing the luciferase reporter assay. As demonstrated in Figure 1C, sequence from wild-type (WT-Luci) 3'-UTR of PLIN5 mRNA was constructed to the downstream of luciferase ORF, as well as its mutated version (mut-Luci). Then, these two constructs were transfected separately into both HT-29 and SW480 cells, along with miR-4284 mimic or miRNA negative control. The activity of the luciferase reporter was examined. It was consistently observed that activity of WT-Luci was dramatically reduced by miR-4284 to $\sim 30 \%$ of the control, in both HT-29 and SW480 cells (Figure 1D). Whereas activity of mut-Luci remained mostly unaffected in miR-4284 transfected cells when compared to the control. Therefore, the complementary sequence within PLIN5 3'-UTR was indeed directly targeted by miR-4284.

\section{MiR-4284 inhibits PLIN5 expression in colon cancer cells}

Next, we tested whether miR-4284 indeed targets PLIN5 in vivo. Endogenous PLIN5 mRNA level in normal human colon cell line CCD-18Co was found to be up-regulated compared with that in HT-29 and SW480 cells (Figure 2A). Next, both HT-29 and SW480 cells were transfected with miR-4284 as well as negative control, followed by qRT-PCR to assess the transcript level of PLIN5. Levels of miR-4284 in HT-29 and SW480 cells were, as expected, highly up-regulated upon transfection (Figure 2B). In comparison with the control, miR-4284 greatly reduced PLIN5 mRNA levels in both lines of colon cancer cells (Figure 3A), evidently demonstrating that PLIN5 was a bona fide target of miR-4284. With antibody against PLIN5, we verified that PLIN5 protein was drastically
A

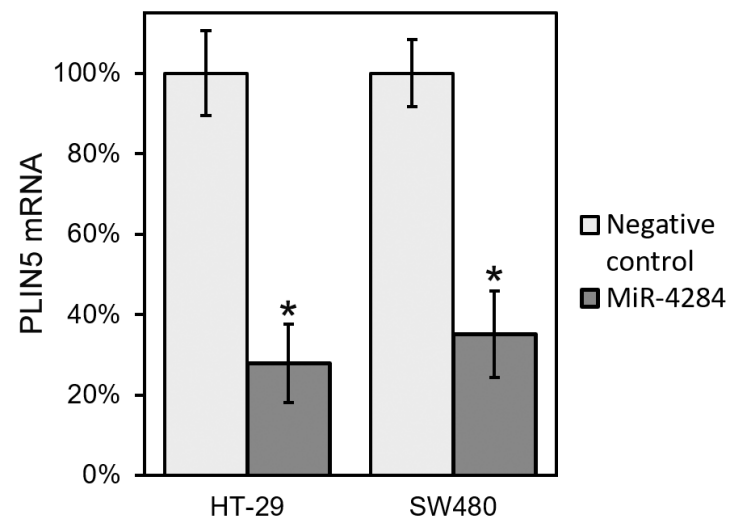

B

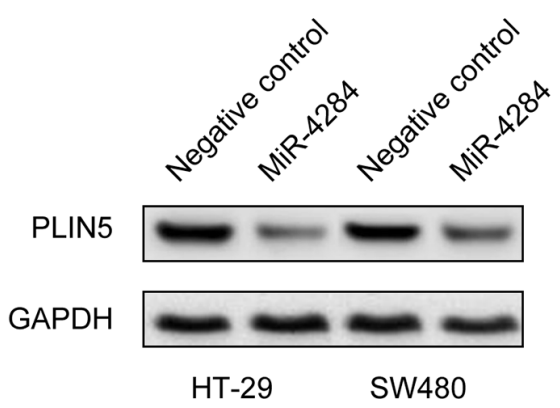

HT-29

Figure 3. PLIN5 is down-regulated by miR-4284 expression in colon cancer cell lines. Levels of PLIN5 mRNA (A) and protein (B) in HT-29 and SW480 cells transfected with either miR-4284 or miRNA negative control. Values were mean \pm SD of 3 independent biological replicates; ${ }^{*} \mathrm{P}<0.05$, compared to negative control. 
A

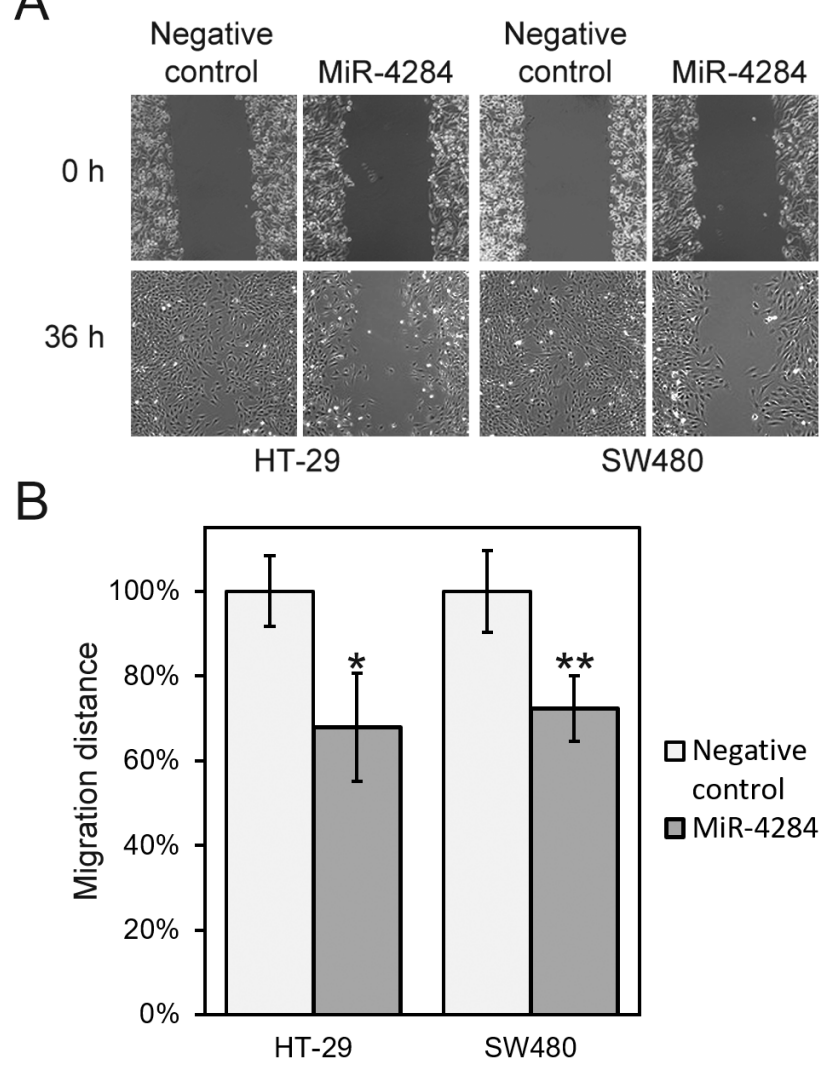

C

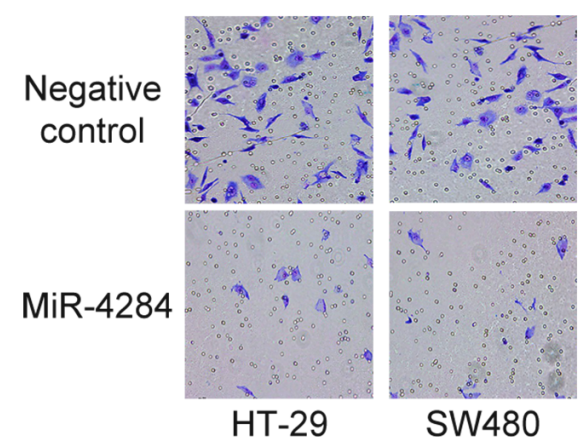

$\mathrm{D}$

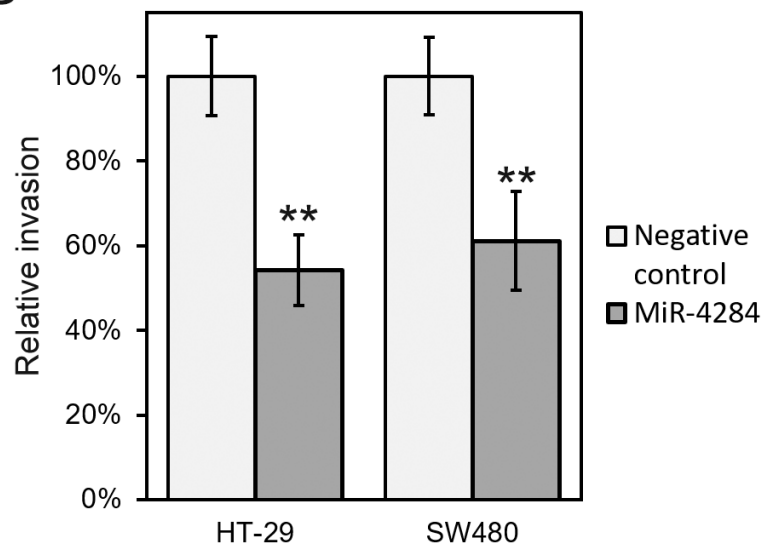

Figure 4. MiR-4284 expression suppresses migration and invasion of colon cancer cells. (A) Wound-healing assay for HT-29 and SW480 cells, transfected with either miR-4284 or negative control. (B) The migrated distances relative to respective controls at $36 \mathrm{~h}$ after the wound being generated were measured. (C) Cell invasion assay for HT-29 and SW480 cells, transfected with either miR-4284 or miRNA negative control. (D) The amounts of invaded cells relative to the respective control were calculated $24 \mathrm{~h}$ after seeding. Values were mean \pm SD of 3 independent biological replicates; ${ }^{* *} \mathrm{P}<0.01$, * $\mathrm{P}<0.05$, compared with negative control.

decreased as well in cells transfected with miR-4284, but not the negative control (Figure 3B).

\section{MiR-4284 inhibits migration and invasion of colon cancer cells}

In order to elucidate the role of miR-4284 in colon cancer tumorigenesis, we evaluated the wound-healing capacity and the invasive properties of miR-4284 or control miRNA transfected HT-29 and SW480 cells. Indeed, the migration rates of both cell lines were greatly reduced after miR-4284 transfection in comparison to control experiments (Figure 4A and 4B). Further, miR4284 transfection also lowered the invasion rates of both HT-29 and SW480 cells (Figure 4C and 4D). Altogether, these data strongly indicated the anti-tumor property of miR-4284 in colon cancer.

\section{MiR-4284 reverses the epithelial-mesenchymal transition (EMT) of colon cancer cells}

As we were performing the aforementioned assays, we frequently observed morphological changes in cells when they were transfected with miR-4284. Both HT29 and SW480 cells originally displayed an elongated fibroblastoid morphology; however, upon miR-4284 transfection both lines of cells assumed a rounded cell shape (data not shown), which suggested a loss of EMT phenotype. To examine this possibility, we assessed attachment and detachment capacities of both lines of colon cancer cells following transfection with miR4284 or the negative control. We found that miR-4284 dramatically lowered both attachment and detachment rates of HT-29 and SW480 cells (Figure 5A and 5B). These findings indicated an inhibition of EMT characteristics at the level of cell morphology. We further assessed if the same trend still held at the molecular level. In this context, we examined the transcript levels of E-cadherin (epithelial marker), N-cadherin and Vimentin (both mesenchymal markers). Consistently, miR-4284 reduced E-cadherin, while up-regulated $\mathrm{N}$-cadherin and Vimentin, in comparison with control transfected cells (Figure 5C), suggesting reversed EMT phenotype at the protein level.

\section{Discussion}

In our current study, we showed that miR-4284 was suppressed in HT-29 and SW480 cells, both human colon cancer cell lines, with CCD-18Co cells (normal colon cell line) as the control. Interestingly, through in silico analysis, we found a putative binding site of miR-4284 on the 3'-UTR of PLIN5 mRNA. Through luciferase reporter assay, we determined that the 3'-UTR sequence was directly targeted by miR-4284. Moreover, introduction of 
A

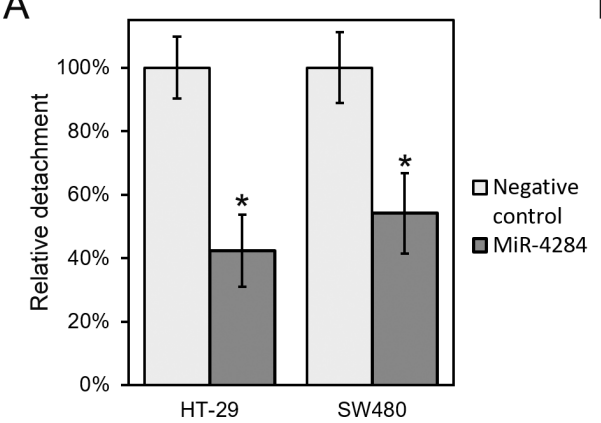

B

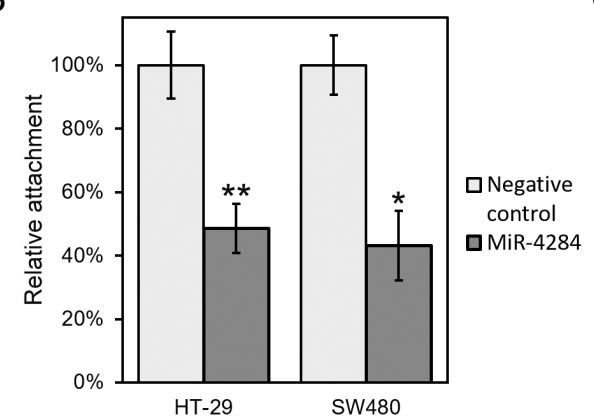

C

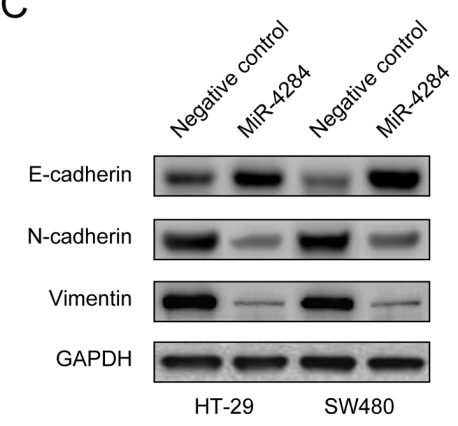

Figure 5. MiR-4284 inhibits epithelial-mesenchymal transition (EMT) of colon cancer cells. (A) Cell attachment and (B) detachment assays were conducted in HT-29 and SW480 cells transfected with either miR-4284 or miRNA negative control. Values were mean \pm SD of 3 independent biological replicates; ${ }^{* *} \mathrm{P}<0.01,{ }^{*} \mathrm{P}<0.05$, compared to negative control. (C) Expression of EMT phenotypic proteins was assessed in HT-29 and SW480 cells transfected with either miR-4284 or miRNA negative control.

miR-4284 into HT-29 and SW480 cells down-regulated PLIN5 at both transcript and protein levels, indicating that PLIN5 was repressed by miR-4284 in vivo. Further, miR4284 expression dramatically inhibited the migratory and invasive activities of colon cancer cells, likely through reverse of EMT, as indicated by a substantial reduction in E-cadherin expression (epithelial marker) and an elevation in the expression of N-cadherin as well as Vimentin (both mesenchymal markers).

EMT refers to the vital process for differentiated epithelial cells to change status, acquiring the capacity to invade, resist apoptosis, and disseminate, which eventually leads to metastasis formation $(17,18)$. MiRNAs are frequently associated with the EMT of multiple human cancers, colon cancer included, by altered expression of tumor suppressor genes or oncogenes (19). For instance, in lung cancer, miR-134/487b/665 cluster affects EMT mediated by transforming growth factor- $\beta$ (TGF- $\beta$ ) via membrane associated guanylate kinase inverted 2 (MAGI2) (20). In addition, in breast cancer miR-125b/489 reportedly regulates EMT $(21,22)$. Down-regulated miR200 c contributes to epidermal growth factor receptor (EGFR) inhibitor resistance through manifestation of EMT features (23). Further, miR-216a/217 was demonstrated to target SMAD7 and PTEN to induce EMT in liver cancer (24).

A recent study has identified co-regulated miRNAs with the potential to cooperate during the EMT process, by combined computational and experimental approaches (25). This study reported that miRNAs could serve as a secondary regulatory layer post transcription, enhancing transcriptional modulations on EMT-associated processes (i.e., cell-adhesion or organization of extracellular matrix) while at the same time dampening transcriptional impacts on irrelevant genes. This discovery raises significant concern over the function of miRNAs in the metastasis of cancer. In line with this recent discovery, we hereby report that a novel miR-4284 is significantly down-regulated and directly targets PLIN5 to inhibit the EMT process in colon cancer cells. Ectopic expression of miR-4284 dramatically reduced endogenous PLIN5 mRNA and protein. In addition, miR-4284 also functioned to suppress the migration, invasion and EMT of colon cancer cells. PLIN5 belongs to the PAT (perilipin, adipophilin and TIP47) family of amphiphilic proteins, the main structural proteins associated with lipid droplet fractions (26). To date, the major function of PLIN5 is mainly to maintain lipid homeostasis by inhibiting lipolysis (27), and report on the involvement of PLIN5 in human cancer is limited. A recent investigation found that PLIN5 was robustly expressed in tumors of human patients with hepatocellular carcinoma (28). Results of our current study have brought this discovery one step further, by identifying its upstream regulator, miR-4284, whose expression directly suppresses PLIN5 in colon cancer cells. Our future study will focus on verifying the involvement of PLIN5 in colon cancer using an animal model in vivo.

In summary, this study presented the first data involving miR-4284 and PLIN5 in colon cancer EMT. EMT is commonly believed to correlate with the aggressiveness of cancer cells, for instance capacities to migrate and invade, hence reverse or inhibition of such transition may inspire new therapeutic strategies for cancer treatments. To this end, we discovered that miR-4284 reversed the EMT of colon cancer cell lines HT-29 and SW480, which was likely the reason miR-4284 could potently inhibit the migratory and invasive activities of colon cancer cells. Hence, our results highlight the potential of miR4284 as a promising target in colon cancer treatment, via inhibiting the expression of PLIN5 and EMT process of colon cancer cells.

\section{Conflict of interest}

The authors declare that they have no conflicts of interest to disclose.

\section{Funding}

This work was funded by Wuxi Taihu Lake talent project (2020THRC-GD-5); Wuxi Medical Emphasis Disciplines Funding (syxzdxk-pwk); Wuxi Science and Technology Development Plan (CZE02H1701); Top Talent Support Program for young and middle-aged people of Wuxi Health Committee (BJRC-9); Youth Science Foundation Project of Wuxi health and Health Committee (Q202048). 


\section{References}

1. Siegel R, Desantis C, Jemal A. Colorectal cancer statistics 2014. CA Cancer J Clin. 2014;64(2):104-17

2. Arnold M, Sierra MS, Laversanne M, Soerjomataram I, Jemal A, Bray F. Global patterns and trends in colorectal cancer incidence and mortality. Gut. 2017;66(4):683-91.

3. Guinney J, Dienstmann R, Wang X, de Reynies A, Schlicker A, Soneson C, et al. The consensus molecular subtypes of colorectal cancer. Nat Med. 2015;21(11):1350-6.

4. Bartel DP. MicroRNAs: genomics, biogenesis, mechanism and function. Cell. 2004;116(2):281-97.

5. Lee RC, Feinbaum RL, Ambros V. The C. elegans heterochronic gene lin-4 encodes small RNAs with antisense complementarity to lin-14. Cell. 1993;75(5):843-54

6. Reinhart BJ, Slack FJ, Basson M, Pasquinelli AE, Bettinger $\mathrm{JC}$, Rougvie AE, et al. The 21-nucleotide let-7 RNA regulates developmental timing in Caenorhabditis elegans. Nature. 2000;403(6772):901-6

7. Pasquinelli $A E$, Reinhart BJ, Slack F, Martindale $M Q$, Kuroda $\mathrm{MI}$, Maller B, et al. Conservation of the sequence and temporal expression of let-7 heterochronic regulatory RNA. Nature. 2000;408(6808):86-9.

8. Esteller M. Non-coding RNAs in human disease. Nat Rev Genet. 2011;12(12):861-74.

9. Iorio MV, Croce CM. MicroRNA dysregulation in cancer: diagnostics, monitoring and therapeutics. A comprehensive review. EMBO Mol Med. 2012;4(3):143-59.

10. Rupaimoole R, Calin GA, Lopez-Berestein G, Sood AK. miRNA deregulation in cancer cells and the tumor microenvironment. Cancer Discov. 2016;6(3):235-46

11. Bader AG. miR-34 - a microRNA replacement therapy is headed to the clinic. Front Genet. 2012;3:120.

12. Li $Y$, Shen $Z$, Jiang $H$, Lai $Z$, Wang $Z$, Jiang $K$, et al. MicroRNA4284 promotes gastric cancer tumorigenicity by targeting ten-eleven translocation 1. Mol Med Rep. 2018;17(5):6569-75.

13. Yang F, Nam S, Brown CE, Zhao R, Starr R, Ma Y, et al. A novel berbamine derivative inhibits cell viability and induces apoptosis in cancer stem-like cells of human glioblastoma, via up-regulation of miRNA-4284 and JNK/AP-1 signaling. PLoS One. 2014;9(4):e94443.

14. Munari E, Marchionni L, Chitre A, Hayashi M, Martignoni G, Brunelli $M$, et al. Clear cell papillary renal cell carcinoma: micro-RNA expression profiling and comparison with clear cell renal cell carcinoma and papillary renal cell carcinoma. Hum Pathol. 2014;45(6):1130-8.

15. McDermott N, Meunier A, Wong S, Buchete V, Marignol L. Profiling of a panel of radioresistant prostate cancer cells identifies deregulation of key miRNAs. Clin Transl Radiat Oncol. 2017:2:63-8.

16. Agarwal V, Bell GW, Nam JW, Bartel DP. Predicting effective
microRNA target sites in mammalian mRNAs. Elife. 2015;4.

17. Gregory PA, Bert AG, Paterson EL, Barry SC, Tsykin A Farshid G, et al. The miR-200 family and miR-205 regulate epithelial to mesenchymal transition by targeting ZEB1 and SIP1. Nat Cell Biol. 2008;10(5):593-601.

18. Hanahan D, Weinberg RA. Hallmarks of cancer: the next generation. Cell. 2011;144(5):646-74.

19. Wang Z, Li Y, Ahmad A, Azmi AS, Kong D, Banerjee S et al. Targeting miRNAs involved in cancer stem cell and EMT regulation: An emerging concept in overcoming drug resistance. Drug Resist Updat. 2010;13(4-5):109-18.

20. Kitamura K, Seike M, Okano T, Matsuda K, Miyanaga A, Mizutani $\mathrm{H}$, et al. MiR-134/487b/655 cluster regulates TGF-beta-induced epithelial-mesenchymal transition and drug resistance to gefitinib by targeting MAGI2 in lung adenocarcinoma cells. Mol Cancer Ther. 2014;13(2):444-53.

21. Jiang $L$, He D, Yang D, Chen Z, Pan Q, Mao A, et al MiR-489 regulates chemoresistance in breast cancer via epithelial mesenchymal transition pathway. FEBS Lett. 2014;588(11):2009-15.

22. Wu YM, Chen ZJ, Liu H, Wei WD, Lu LL, Yang XL, et al Inhibition of ERRalpha suppresses epithelial mesenchymal transition of triple negative breast cancer cells by directly targeting fibronectin. Oncotarget. 2015.

23. Shien K, Toyooka S, Yamamoto H, Soh J, Jida M, Thu KL, et al. Acquired resistance to EGFR inhibitors is associated with a manifestation of stem cell-like properties in cancer cells. Cancer Res. 2013;73(10):3051-61.

24. Xia H, Ooi LL, Hui KM. MicroRNA-216a/217-induced epithelial-mesenchymal transition targets PTEN and SMAD7 to promote drug resistance and recurrence of liver cancer. Hepatology. 2013;58(2):629-41

25. Cursons J, Pillman KA, Scheer KG, Gregory PA, Foroutan $M$, Hediyeh-Zadeh $S$, et al. Combinatorial targeting by microRNAs co-ordinates post-transcriptional control of EMT. Cell Syst. 2018;7(1):77-91 e7.

26. Kimmel AR, Brasaemle DL, McAndrews-Hill M, Sztalryd C, Londos C. Adoption of PERILIPIN as a unifying nomenclature for the mammalian PAT-family of intracellular lipid storage droplet proteins. J Lipid Res. 2010;51(3):46871.

27. Yamaguchi T, Matsushita S, Motojima K, Hirose F, Osumi T. MLDP, a novel PAT family protein localized to lipid droplets and enriched in the heart, is regulated by peroxisome proliferator-activated receptor alpha. J Biol Chem. 2006;281(20):14232-40.

28. Asimakopoulou A, Vucur M, Luedde T, Schneiders S, Kalampoka S, Weiss TS, et al. Perilipin 5 and lipocalin 2 expression in hepatocellular carcinoma. Cancers (Basel). 2019;11(3). 